\section{Case Reports in Ophthalmology}

\title{
In vivo Confocal Microscopy in Differentiating Ipilimumab-Induced Anterior Uveitis from Metastatic Uveal Melanoma
}

\author{
Hayyam Kiratli Mehmet C. Mocan Murat İrkeç \\ Ocular Oncology Service, Department of Ophthalmology, Hacettepe University School of \\ Medicine, Ankara, Turkey
}

\section{Keywords}

Ipilimumab · T-cell $\cdot$ Uveitis · Uveal melanoma $\cdot$ Confocal microscopy

\begin{abstract}
This report aims to describe the facilitating role of in vivo confocal microscopy in differentiating inflammatory cells from a metastatic process in a patient with uveal melanoma and multiple systemic metastases who developed anterior uveitis while under ipilimumab treatment. A 43-year-old woman developed systemic metastases 11 months after treatment of amelanotic choroidal melanoma in her right eye with $30 \mathrm{~Gy}$ fractionated stereotactic radiotherapy. She first received temozolomide and then 4 cycles of ipilimumab $3 \mathrm{mg} / \mathrm{kg} / \mathrm{day}$. After the third cycle, severe anterior uveitis with coarse pigment clumps on the lens was seen in the left eye. Her left visual acuity declined from 20/20 to 20/80. Confocal microscopy revealed globular keratic precipitates with hyperreflective inclusions and endothelial blebs all suggestive of granulomatous uveitis. The uveitic reaction subsided after a 3-week course of topical corticosteroids, and her visual acuity was $20 / 20$ again. Although uveal melanoma metastatic to the intraocular structures of the fellow eye is exceedingly rare and metastasis masquerading uveitis without any identifiable uveal lesion is even more unusual, it was still mandatory to rule out this distant possibility in our particular patient who already had widespread systemic metastases. Confocal microscopy was a useful complementary tool by identifying the inflammatory features of the keratic precipitates.




\section{Case Reports in Ophthalmology}

Kiratli et al.: In vivo Confocal Microscopy in Differentiating Ipilimumab-Induced Anterior Uveitis from Metastatic Uveal Melanoma

\section{Introduction}

Ipilimumab is a human monoclonal immunoglobulin G1 antibody with a plasma half-life of 12-14 days and targets the cytotoxic T-lymphocyte-associated antigen 4 (CTLA-4) receptors $[1,2]$. The transiently expressed CTLA-4 on activated T-cells acts as a negative regulator and transmits inhibitory signals resulting in downregulation of T-cell functions and maintenance of peripheral tolerance [3]. Studies have demonstrated that the use of the T-cell potentiator ipilimumab was associated with a significant improvement in the overall survival of patients with metastatic melanoma [2]. However, the ipilimumab-enhanced T-cell activation may also frequently cause colitis, hepatitis, dermatitis and uveitis, as important side effects [1].

We herein report the case of a patient with choroidal melanoma and widespread systemic metastases who developed atypical anterior uveitis in the fellow eye while under ipilimumab treatment. In vivo confocal microscopy (IVCM) findings allowed differentiation from metastatic melanoma, which was a potential diagnostic possibility in this patient.

\section{Materials and Methods}

The medical records, the results of imaging studies including magnetic resonance imaging and IVCM and the clinical course with treatment outcomes were reviewed.

A 43-year-old woman presented with a 3-week history of gradual loss of vision in the right eye. Her best corrected vision was counting fingers in the right eye and 20/20 in the left eye. Fundus examination showed a nasal juxtapapillary amelanotic melanoma measuring $11 \times 10 \mathrm{~mm}$ in basal dimensions and $8.6 \mathrm{~mm}$ in thickness (fig. 1). There was associated serous retinal detachment involving the inferior half of the retina. The rest of the ocular examination was noncontributory. A systemic workup including liver function tests and thoracoabdominal MRI were normal. The right eye was treated with fractionated stereotactic radiosurgery (Cyberknife, Accuray, Calif., USA) with a total dose of 30 Gy in 3 sessions. Six months after treatment, the tumor showed partial regression with disappearance of the subretinal fluid. At 11 months, she was discovered to have 3 distinct metastatic foci in the liver, the largest of which measured $4.5 \mathrm{~cm}$ in diameter. Incisional biopsy of one of these lesions demonstrated $B R A F$ mutation-negative metastatic melanoma.

The patient received 3 cycles of temozolomide $250 \mathrm{mg} / \mathrm{m}^{2}$, but the metastatic lesions continued to increase both in size and number. She was then put on 4 cycles of ipilimumab $3 \mathrm{mg} / \mathrm{kg} /$ day, administered as intravenous infusion in $90 \mathrm{~min}$. After the third cycle, she presented with flashes and floaters in the left eye. Her vision was no light perception in the right eye and 20/80 in the left eye. Corneal edema, iris neovascularization and mild vitreous hemorrhage were present in the right eye. Tumor regrowth was also noted. The left eye had large, pigmented keratic precipitates (KP), 2+ pigmented cells in the anterior chamber and anterior vitreous, and large pigment clumps on the anterior surface of the lens (fig. 2). There were no posterior synechia. The posterior segment appeared normal.

IVCM was performed using Confoscan 3.0 (Vigonza, Italy) attached to an immersion lens (Achroplan 40×/0.75 W, Zeiss, Germany). IVCM revealed large, globular-type KP in both eyes. These precipitates had conglomerate morphology and contained multiple hyperreflective inclusions indicative of granulomatous uveitis (fig. 3). Extensive stromal edema of the right eye precluded clear visualization of the endothelial layer (fig. 3a). Multiple globular KP with sizes ranging between 80 and $120 \mu \mathrm{m}$ were detected at the level of the endothelium OS 
(fig. 3b). Several blebs, indicative of active intraocular inflammation, were observed as small empty lacunae between the endothelial cells (fig. $3 \mathrm{~b}$ ).

These results were in favor of anterior uveitis presumably induced by ipilimumab rather than a metastatic event. A 3-week course of topical dexamethasone cleared all the reaction in the anterior chamber and anterior vitreous of the left eye. Her left visual acuity was 20/20 again. In the meantime, the right eye was enucleated. Histopathologically the tumor was predominantly epitheloid cell mixed-type melanoma. After enucleation, new metastatic foci developed in her lungs, bones and spleen. She refused further treatment and expired 3 months later.

\section{Discussion}

Ipilimumab disrupts peripheral tolerance to both tumor and self-antigens and causes mechanism-based autoimmune and inflammatory toxic effects, collectively described as immune-related adverse events, which can be encountered in $72.3 \%$ of patients receiving this drug [4]. Therapy-induced inflammatory infiltrates composed of activated T-cells may also cause growth of existing metastases [4]. The most common ipilimumab-induced immune-related adverse events are maculopapular rash, diarrhea/colitis and hepatitis $[4,5]$. Ocular adverse effects in the form of episcleritis and uveitis develop in less than $1 \%$ of patients taking ipilimumab and usually occur a median of 2 months after the initiation of treatment [4]. These patients may also have associated colitis [6, 7]. Our patient did not have any evidence of gastrointestinal upset. Other less frequent side effects include conjunctivitis, orbital myositis and Tolosa-Hunt syndrome [7]. The first report of uveitis associated with CTLA-4 blockade appeared in 2004, describing 2 patients with anterior uveitis, vitritis and optic nerve head swelling [8]. One of these patients had severe anterior uveitis and coarse iris pigment epithelial clumps on the anterior lens surface, similar to our case [7].

Metastasis of choroidal melanoma to the intraocular structures of the fellow eye is extremely rare and represents only $0.7-1.2 \%$ of all metastatic intraocular tumors [9]. Reported patients typically had a funduscopically identifiable choroidal mass, whereas our case presented with pigmented cells in the anterior chamber and large pigment clumps on the lens surface without a choroidal lesion [9-11]. Still, however, given the presence of widespread systemic metastases, the likelihood of intraocular metastasis was not null.

Confocal microscopy is in use for visualization of infectious agents and inflammatory cells in the cornea and differentiation of corneal dystrophies [12]. This technique allowed identification of the predominant inflammatory cell morphological patterns forming the KP in various conditions associated with granulomatous and nongranulomatous uveitis [1214]. Globular KP, as we observed in our patient, are characterized by multiple hyperreflective inclusions in a larger round cluster and sometimes may have dendritiform extensions $[12,14]$. This type of KP is most often associated with granulomatous uveitis, acute anterior uveitis and Fuchs' uveitis syndrome $[12,14]$. Globular appearance of KP without accompanying dendritiform extension is more suggestive of noninfectious uveitic entities [12,13]. The coexistence of endothelial blebs and the globular structures visualized with IVCM in this patient are highly suggestive of active intraocular inflammation as opposed to a metastatic process $[12,13]$. In addition, a metastatic lesion would be expected to persist on the corneal endothelium and be still visible with IVCM after topical steroid therapy, whereas the corneal lesions in our case resolved following a 3-week course of topical steroid therapy supporting their inflammatory nature. 
Kiratli et al.: In vivo Confocal Microscopy in Differentiating Ipilimumab-Induced Anterior Uveitis from Metastatic Uveal Melanoma

Our case showed that ipilimumab may induce granulomatous anterior uveitis characterized by large, globular KP. The KP in granulomatous uveitis appear as large globular structures containing hyperreflective inclusions measuring between 50 and $150 \mu \mathrm{m}$ in diameter in IVCM $[12,13]$. In contrast, the KP that arise in nongranulomatous uveitic entities are much smaller, in the range of 5-15 $\mu \mathrm{m}$, appear as scattered single dot-like structures and do not interfere with the visualization of the neighboring endothelial cells [12]. Up until the writing of this manuscript, we have not observed metastatic melanoma lesions on the corneal endothelium in any patient and are not aware of any reports in the literature that highlight their characteristics with IVCM. However, we believe that in our patient, in whom a metastatic process was a distant possibility, IVCM proved useful in suggesting the inflammatory nature of these cells.

\section{Statement of Ethics}

The family members have given informed consent agreeing the publication of all the medical information pertaining to the deceased patient subject to this report. The use of ipilimumab was approved by the oncology council at the Oncology Institute, Hacettepe University.

\section{Disclosure Statement}

The authors did not receive any sponsorship or funding related to this report. The authors have no conflicts of interest.

\section{References}

1 Kheir WJ, Sniegowsky MC, El-Sawy T, et al: Ophthalmic complications of targeted cancer therapy and recently recognized ophthalmic complications of traditional chemotherapy. Surv Ophthalmol 2014;59:493-502.

2 Hodi FS, O'Day SJ, McDermott DF, et al: Improved survival with ipilimumab in patients with metastatic melanoma. N Engl J Med 2010;363:711-723.

- Peggs KS, Quezada SA, Korman AJ, Allison JP: Principles and use of anti-CTLA4 antibody in human cancer. Curr Opin Immunol 2006;18:206-213.

-4 Weber JS, Kahler KC, Hauschild A: Management of immune-related adverse events and kinetics of response with ipilimumab. J Clin Oncol 2012;30:2691-2697.

-5 Weber JS, Dummer R, de Pril V, et al: Patterns of onset and resolution of immune-mediated adverse effects of special interest with ipilimumab. Cancer 2013;119:1675-1682.

-6 Papavasileiou E, Prasad S, Freitag SK, Sobrin L, Lobo A-M: Ipilimumab-induced ocular and orbital inflammation - a case series and review of the literature. Ocul Immunol Inflamm 2016;24:140-146.

7 Rousseau A, Labetoulle M: Effets indésirables oculaires des traitements systémiques: mis à jour. J Fr Ophtalmol 2015;38:876-882.

8 Robinson MR, Chan CC, Yang JC, et al: Cytotoxic T lymphocyte-associated antigen 4 blockade in patients with metastatic melanoma. A new cause of uveitis. J Immunother 2004;27:478-479.

-9 Zografos L, Ducrey N, Beati D, et al: Metastatic melanoma in the eye and orbit. Ophthalmology 2003;110:2245-2256.

10 Bourla DH, Young TA: Treatment considerations for primary uveal melanoma with choroidal metastasis to the fellow eye. Semin Ophthalmol 2007;22:175-177.

11 Khetan V, Ambatipudi S, Gopal L: Presumed metastasis of choroidal melanoma to the contralateral choroid. Retin Cases Brief Rep 2010;4:387-389.

12 Mocan MC, Kadayıfçılar S, İrkeç M: Keratic precipitate morphology in uveitic syndromes including Behçet's disease evaluated with in vivo confocal microscopy. Eye 2009;23:1221-1227. 


\section{Case Reports in Ophthalmology}

\begin{tabular}{l|l}
\hline DOI: 10.1159/000448730 & $\begin{array}{l}\text { @ } 2016 \text { The Author(s). Published by S. Karger AG, Basel } \\
\text { www.karger.com/cop }\end{array}$ \\
\hline
\end{tabular}

Kiratli et al.: In vivo Confocal Microscopy in Differentiating Ipilimumab-Induced Anterior Uveitis from Metastatic Uveal Melanoma

13 Wertheim MS, Mathers WD, Planck SJ, et al: In vivo confocal microscopy of keratic precipitates. Arch Ophthalmol 2004;122:1773-1781.

14 Kanavi MR, Soheilian M: Confocal scan features of keratic precipitates in granulomatous versus nongranulomatous uveitis. J Ophthalmic Vis Res 2011;6:255-258.

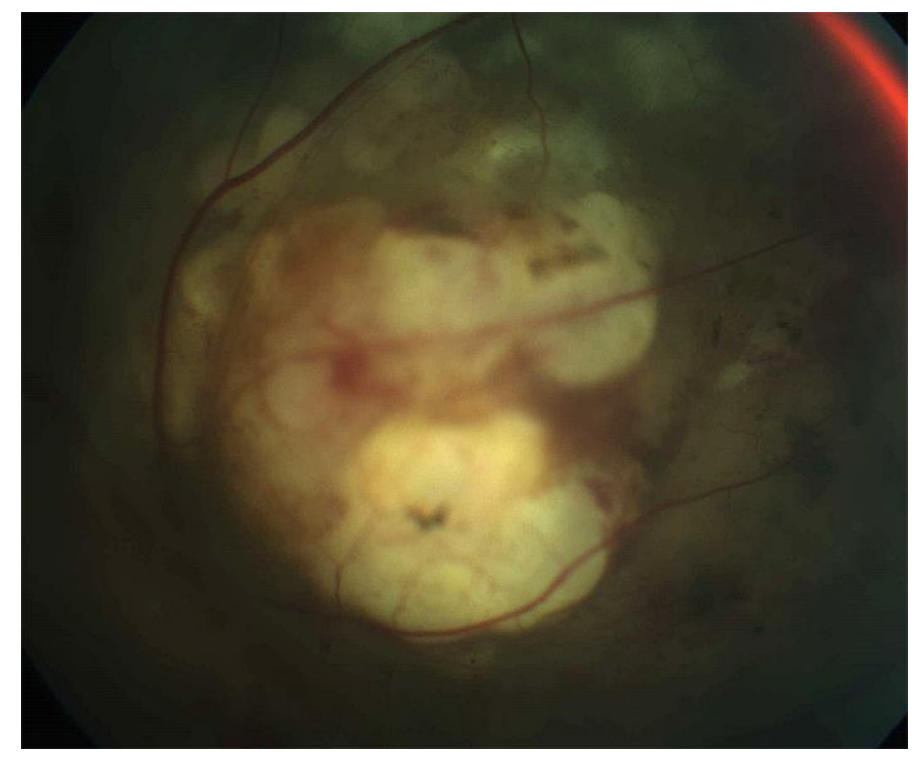

Fig. 1. Amelanotic choroidal melanoma in the right eye before treatment.

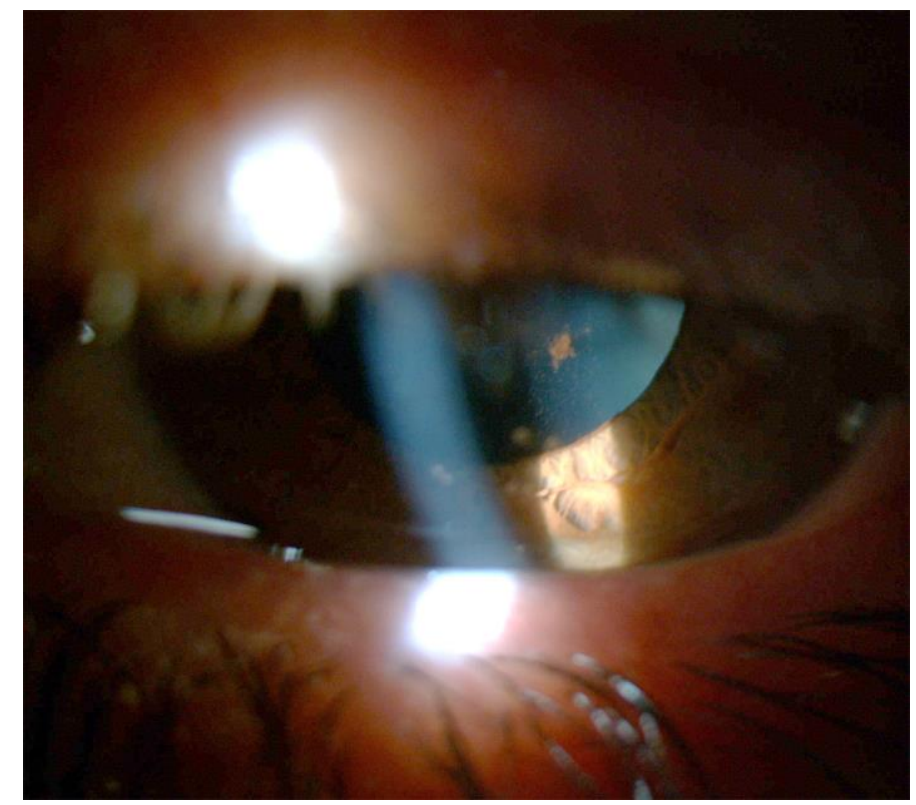

Fig. 2. Slit-lamp view of the left eye showing large pigment agglomerates on the anterior surface of the lens. 
Case Reports in
Ophthalmology

Case Rep Ophthalmol 2016;7:126-131 DOI: $10.1159 / 000448730$

(c) 2016 The Author(s). Published by S. Karger AG, Basel www.karger.com/cop

Kiratli et al.: In vivo Confocal Microscopy in Differentiating Ipilimumab-Induced Anterior Uveitis from Metastatic Uveal Melanoma
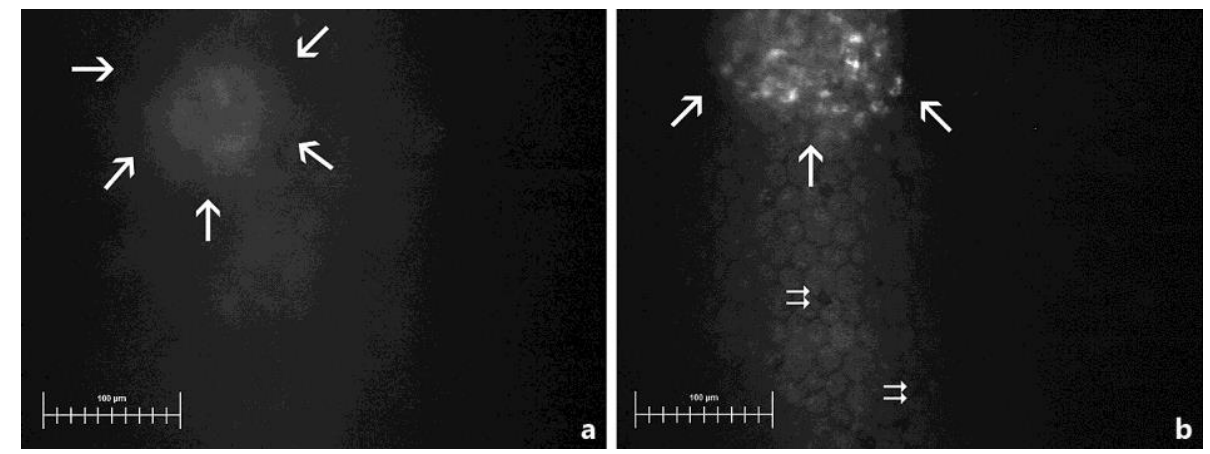

Fig. 3. Confocal microscopy shows faint outline of a globular type of KP in the right eye of the case (a, arrows). Extensive stromal edema was associated with loss of corneal microstructural details. b A large globular type KP with multiple hyperreflective inclusions (large arrows) at the level of endothelium is demonstrated. Several endothelial blebs indicative of active inflammation are also observed as empty lacunae between the cells (double arrows). 